\title{
Grassroots initiatives
}

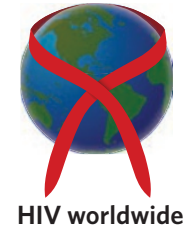

\section{A Cambodian group has developed a pioneering community-based approach to HIV and TB care and research. Amy Maxmen describes how this powerful model is being expanded to other war-torn countries.}

or 30 years, the paediatric ward of the Khmer Soviet Friendship Hospital stood crumbling at the southern end of the Cambodian capital Phnom Penh.

In 2008, supported largely by private donations, the Cambodian Health Committee, a non-governmental organization (NGO), transformed the ward into the Sullivan Outpatient Center for children with HIV and tuberculosis (TB). Later that year, Médecins Sans Frontières (MSF) entrusted the care of 205 HIV-positive children to the centre.

Now called the Global Health Committee (GHC), the Cambodian group was born in 1994 at a refugee camp on the Cambodia-Vietnam border. The genocidal Khmer Rouge regime targeted health-care professionals, so, when its reign ended in 1979, those who survived were left with no medical infrastructure.

Since its inception, the GHC has cared

25,000 people of TB, says Anne Goldfeld, the group's co-founder and professor of medicine at Harvard University. With donors ranging from the drug company Eli Lilly to the United Nations World Food Programme and the US National Institutes of Health, the GHC provides medicines, meals and care for an estimated 15,000 people. The group manages five centres for HIV and TB care and research in Cambodia, stretching from rural provinces to Phnom Penh.

\section{Community involvement}

In 2008, the group expanded its model to Ethiopia. Staff members have also visited refugee camps in Pakistan, and are discussing plans to move into Swaziland and Vietnam. Their approach differs from other missions that deliver care after war, says Paul Hamilton, project director at an aid organization in Uganda, who is not affiliated with the GHC. "I think their commitment is longer and they really work on incorporating the community. And the second thing that is really different is that they do a lot of basic research."
Many international medical NGOs are in the process of leaving Cambodia. At times like these, "the [GHC] becomes more important." says Didier Laureillard, a physician specializing in HIV and TB at the European Georges Pompidou Hospital in Paris, who volunteered with MSF in Cambodia.

The group owes its success in Cambodia in part to sustainability brought about by training and working with the community. Its staff members visit patients at home and enlist local health-care workers to monitor the nutritional quality of meals eaten by those receiving antiretroviral drugs from its clinics.

Goldfeld also initiated basic research in the mid-1990s. Her team is conducting a clinical trial on the ideal time for those co-infected with TB and HIV to begin taking AIDS drugs.
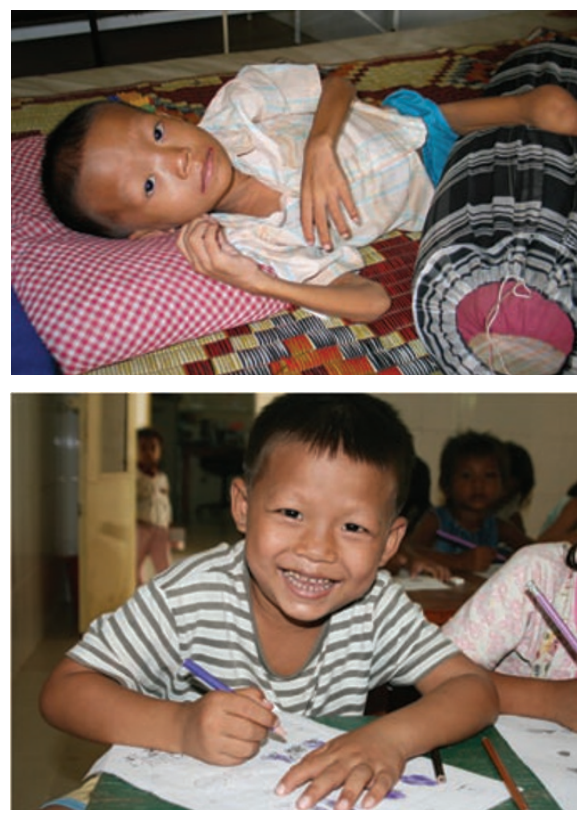

The Cambodian group has cared for many children infected with HIV and tuberculosis, including Ye How (at age six in 2006, top; and after successful treatment a year later, bottom).
"There were a lot of people who doubted our ability to do it because Cambodia had no infrastructure," she says. "Not only did we end up recruiting 661 patients but, what's more, we've enhanced care all over Cambodia for TB and AIDS."

\section{Fighting the brain drain}

Goldfeld has mentored local clinicians in writing proposals, designing studies and publishing results. That training is bound to have a broader impact, says Kristian Olson, a clinician educator at Massachusetts General Hospital and health advisor for the GHC.

"If you create this cadre of inquisitive physician scientists that are driven to answer compelling questions, you'll do a lot for staff retention and reverse the brain drain," Olson says.

Olson volunteers with GHC's outpost in Addis Ababa, set up with the Ethiopian Ministry of Health's approval, to combat HIV and TB. According to a 2008 World Health Organization report on Ethiopia, $40 \%$ of TB patients tested there were HIV-positive.

The Cambodian team has repeatedly visited Addis Ababa to train local doctors. Last year, with funding from the Jolie-Pitt Foundation, it opened the Zahara Children's Center for HIV-positive kids modelled after the group's similar Maddox Chivan Children's Center in Cambodia.

With some tweaks, the GHC's model for building medical infrastructure will work in any impoverished, war-torn country, says Sok Thim, a former Cambodian refugee and the group's co-founder. "We have the model and the means to deliver it," Thim says, "We want to help wherever there is great need for TB care."

Extending models to other countries is harder than it sounds, cautions Hamilton. "I've seen good models work in one country but not transfer to another." For example, he says, the Ethiopian government is wary of NGOs. Yet those affiliated with the GHC say it has succeeded in Ethiopia partly because it works in partnership with the Ministry of Health.

As a survivor of the killing fields, poverty and TB, Thim relates to the frustrations of Ethiopian health-care workers, Olson says.

"Care providers in Addis Ababa see Thim as a medical expert who easily could have been the poor refugee," says Olson. After training with Thim, Olson says, "I heard them going from no confidence to feeling like they will be [part of ] a centre of excellence".

Amy Maxmen is a freelance writer in New York City. 